\title{
Cases of Susac syndrome misdiagnosed with multiple sclerosis
}

\begin{abstract}
Susac syndrome is an autoimmune microvascular endotheliopathy that decrements in visual acuity, diminished hearing, and encephalopathy symptoms. Patients with Susac syndrome are often misdiagnosed with multiple sclerosis because of the difference in symptoms and involving of corpus callosum damage. Here we described some clinical cases of Susac syndrome, provisionally diagnosed as multiple sclerosis.
\end{abstract}

Keywords: susac syndrome, multiple sclerosis, corpus callosum
Volume 9 Issue 6 - 2019

\author{
Natalia V Polekhina,' Zoya V Surnina, ${ }^{2}$ \\ Rodion N Konovalov, ${ }^{3}$ Maria N Zakharova ${ }^{4}$ \\ 'Moscow inter-area division of Multiple Sclerosis, Russia \\ ${ }^{2}$ Employee of Scientific Research Laboratory of new laser \\ technology of Research Institute of Eye Diseases, Russia \\ ${ }^{3}$ Senior Research Scientist of Radiology department of Research \\ Center of Neurology, Russia \\ ${ }^{4}$ Prof., the Chief of 6th department of Research Center of \\ Neurology, Russia
}

Correspondence: Natalia V Polekhina, MD, Moscow inter-area division of Multiple Sclerosis, Moscow, Russia. I27015, Moscow, Piscovaya st., 10. Email natalie.polekhin@gmail.com

Received: December 13, 2019 | Published: December 23, 2019

\section{Introduction}

Susac syndrome (SS) is an autoimmune microvascular endotheliopathy, which results because of the affection of microvascular of the corpus callosum, retinal arterial branches, and arteria auditiva. There are many theories of SS pathogenesis. One of them is that antiendothelial cell antibodies provocate the endothelial cell injury by following the swelling of endothelial cells. It leads to thrombosis and ischemic tissue changes. Depending on what kind of tissue is damaged, it resulting in a decrement in visual acuity, diminished hearing, and encephalopathy. Because of the dissemination of symptoms and corpus callosum damage, this illness frequently is misdiagnosed with multiple sclerosis.

Otherwise, we described some cases of SS.

\section{Case report I}

A 55-year-old male presented in the Research Center of Neurology with temporal upper quadrantanopia at oculus dexter (OD) at the beginning of 2018. At the August 2017, he had an episode of vestibular dysfunction and blurred vision extended a couple of days. Symptoms decreased without treatment. There were no brain MRI lesions at that time. At the end of September, the patient presented a 10-minutes long dysarthria attack.

At the end of October, acute bilateral sensorineural hearing dysfunction happened. Per os dexamethasone treatment and tympanum shunting (on right ear) led to the absence of improvement.

At the beginning of the 2018 temporal upper quadrantanopia, OD was presented. Because of the affection in several parts of the central neural system (CNS) and lesions in the corpus callosum (CC) in MRI, the patient was diagnosed as multiple sclerosis (MS). The neurological exam finds no abnormalities except for visual and hearing problems. The spinal fluid test of oligoclonal antibodies was negative, also as an infection test. Visual evoked potentials were normal. Because of the classical tirade of symptoms, we thought about possible SS. Repeated MRI showed "snowball" lesions in the CC (Figure 1).

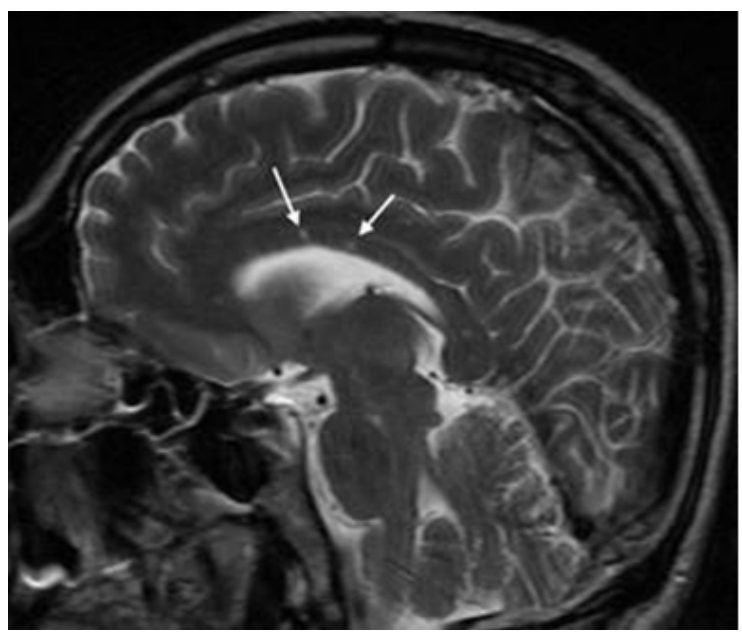

Figure I MRI brain, case I: sagittal T2 sequence, showing multiple hyperintense lesions in the CC (arrows).

Another obligatory analysis in SS is fluorescent angiography of the retina. It showed branch retinal artery occlusion (BRAO). Optical coherence tomography showed no changes in the retinal structure. Owing to anamnesis, clinical picture and test result definitively diagnosis was SS. After the methylprednisolone pulse-therapy (6000 $\mathrm{mg}$ ) patient notices the hearing improvement. Therapy was prolonged by prednisone per os. Also, because of the intensity of symptoms, 
treatment was added by intravenous immunoglobulin (IVIG) and rituximab monoclonal antibody. By now the patient has no relapses of the disease.

\section{Case report 2}

A 27-year-old female presented a one-year history of psychiatric and cognitive symptoms and headache. Then the vision acceded, but there was no hearing dysfunction. Cerebro-spinal fluid and hematology tests were within the normal range. MRI showed "snowball" and "icicle" lesions in the CC (Figure 2). There were BRAO on fluorescent angiography. Long-term high-dose dexamethasone therapy leads to clinical improvement.

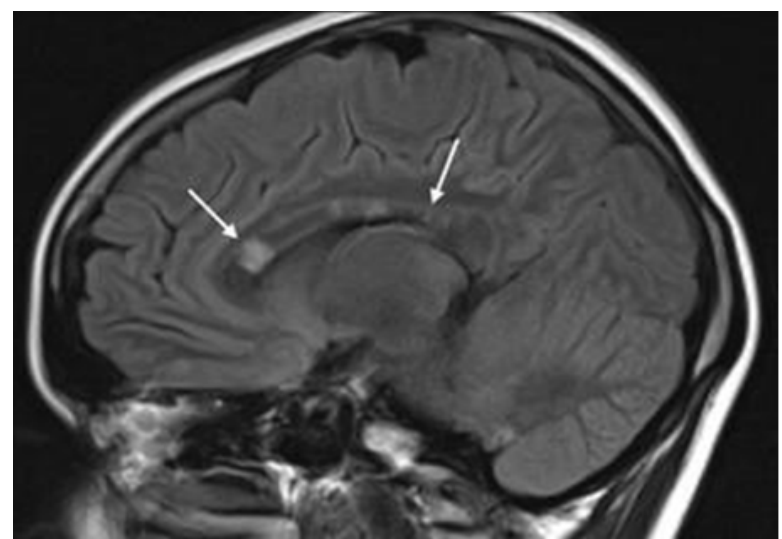

Figure 2 MRI brain, case 2: sagittal T2 sequence, showing multiple hyperintense "snowball" and "icicle" lesions in the CC (arrows).

\section{Case report 3}

A 63-year-old female had a long history of visual loss and mental affection. The MRI showed all classical SS CC lesions (Figure 3 ). Unfortunately, because of the absence of treatment for years prednisolone therapy was ineffective.

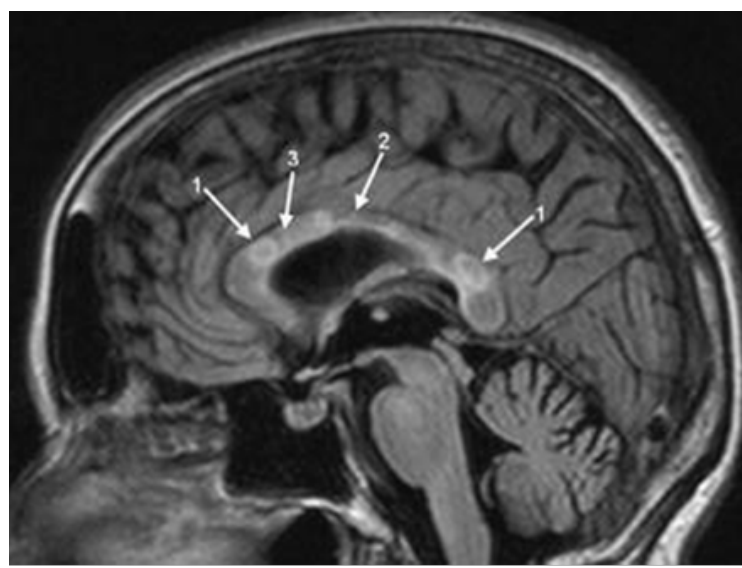

Figure 3 MRI brain, case 3: sagittal T2 sequence, showing "snowball” (I), "needle" (2) and "icicle" (3)lesions in the CC (arrows).

\section{Discussion}

\section{MRI}

Lesions in the $\mathrm{CC}$ are important radiological clues to the diagnosis of MS but may also occur in other neuroinflammatory and nonneuroinflammatory conditions, especially multiple sclerosis. All our cases primary were misdiagnosed as MS. An understanding of the appearance and location of lesions in the CC is important not only for accurate diagnosis and treatment. It also provides insights into pathogenesis.

When the MS diagnosis is suspected, important clues can be obtained from MRI with attention to the inner surface of the CC as this region may be abnormal on MRI for $55 \%$ to $95 \%$ of patients with MS. ${ }^{1,2}$ The ovoid lesions in CC in MS patients are known as Dawson's fingers (Figure 4). Ovoid lesions from the ventricular surface of the callosum into the pericallosal white matter appear to correspond to the pathological areas of perivenular inflammation known as Dawson's fingers.

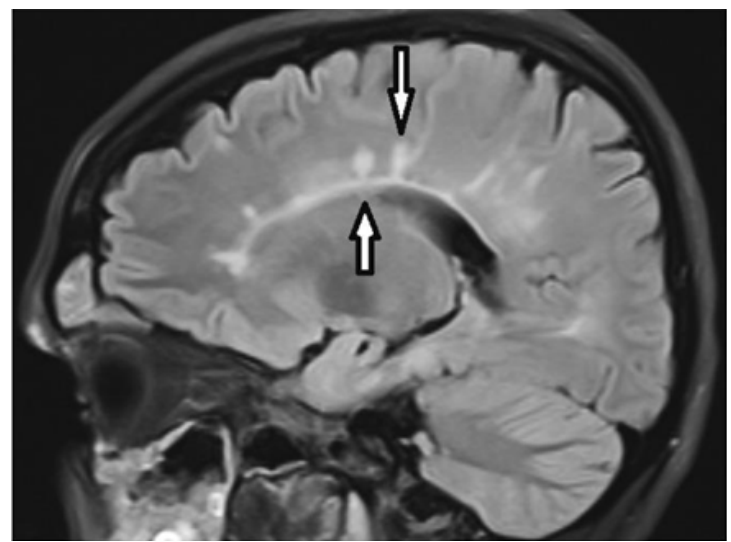

Figure 4 Sagittal MRI FLAIR image of the patient with MS shows hyperintensities perpendicular to the corpus callosum (arrows), so-called Dawson fingers. FLAIR = fluid-attenuated inversion recovery [3].

The hallmark callosal lesion in SS is the "snowball" lesion as the appearance resembles a snowball on sagittal T2 and FLAIR sequences (as you can see in our patients' MRI). These likely pathognomonic lesions of SS are small (3-7 $\mathrm{mm}$ ) and multifocal, involving the central fibers of the $\mathrm{CC}$ with relative sparing of the periphery. ${ }^{1}$ They represent microinfarctions due to occlusion of small precapillary arterioles consistent with the microvascular blood supply of the CC. The roof of the $\mathrm{CC}$ is more commonly affected, with microinfarcts taking on "icicle" and "spoke" configurations (Figure 3). It is important to note that MRI lesions in SS can disappear in comparison to MS lesions (Figure 5).

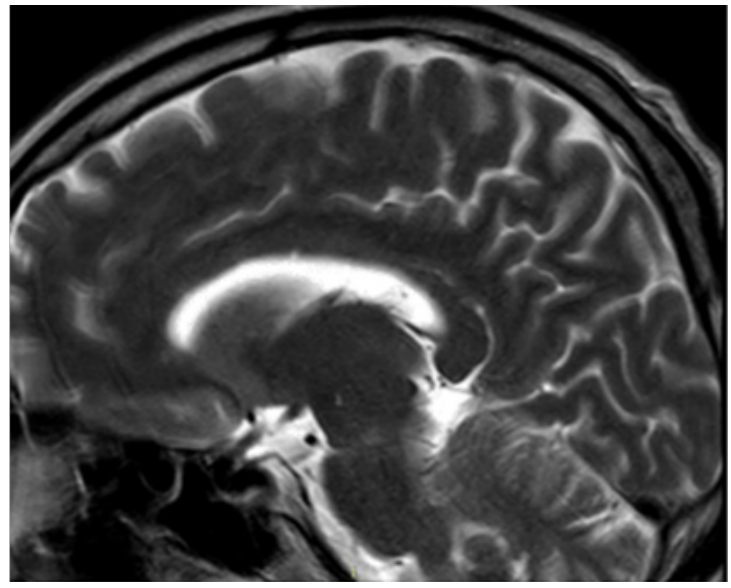

Figure 5 Dynamic MRI, case I: sagittal T2 sequence, showing CC without previous lesions. 


\section{Treatment}

There were no randomized controlled trials in treatment for SS. The treatment has been empirical and based on the autoimmune hypothesis. ${ }^{4,5}$ The mainstay of treatment of SS is corticosteroid (pulse methylprednisolone, followed by high-dose oral prednisone), IVIG, one or two additional immunomodulating drugs (methotrexate, mycophenolate, tacrolimus, or cyclophosphamide, or a combination of two of these), and rituximab. ${ }^{6-8}$

\section{Conclusion}

SS characterized by the clinical triad of encephalopathy, visual and hearing dysfunction. MRI plays a big part in diagnostics because of pathognomonic CC lesions. Early start of prednisolone and immunomodulating treatment leads to a favorable prognosis.

\section{Acknowledgments}

None.

\section{Conflicts of interest}

The author declares no conflicts of interest.

\section{Funding}

None.

\section{References}

1. Garg N, Reddel SW, Miller DH, et al. The corpus callosum in the diagnosis of multiple sclerosis and other CNS demyelinating and inflammatory diseases. J Neurol Neurosurg Psychiatry. 2015;86(12):1374-1382.

2. Gean-Marton AD, Vezina LG, Marton KI, et al. Abnormal corpus callosum: a sensitive and specific indicator of multiple sclerosis. Radiology. 1991;180(1):215-221.

3. Matthews L, Marasco R, Jenkinson M. Distinction of seropositive NMO spectrum disorder and MS brain lesion distribution. J Neurology. 2013;80(14):1330-1337.

4. Rennebohm RM, Asdaghi N, Srivastava S, et al. Guidelines for treatment of Susac syndrome - An update. Int J Stroke. 2018.

5. Rennebohm RM1, Lubow M, Rusin J, et al. Aggressive immunosuppressive treatment of Susac's syndrome in an adolescent: using treatment of dermatomyositis as a model. Pediatr Rheumatol Online J. 2008;6:3.

6. Dalakas MC, Illa I, Dambrosia JM, et al. A controlled trial of highdose IVIG infusions as treatment for dermatomyositis. $N$ Engl J Med. 1993;329(27):1993-2000.

7. Oddis CV, Reed AM, Aggarwal R, et al. Rituximab in the treatment of refractory adult and juvenile dermatomyositis and adult polymyositis: a randomized, placebophase trial. Arthritis Rheum. 2013;65(2):314-324.

8. Palmer S, Bradley WG, Chen DY, et al. Subcallosal striations: early findings of multiple sclerosis on sagittal, thin-section, fast FLAIR MR images. Radiology. 1999;210(1):149-153. 